Military Technical College

Kobry El-Kobba

Cairo, Egypt

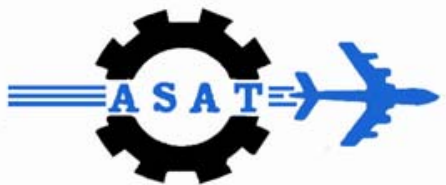

12-th International Conference

on

Aerospace Sciences \&

Aviation Technology

\title{
MODELLING TECHNIQUES ASSESSMENT AND OPTIMIZATION OF LAMINATED AND SANDWICH COMPOSITES UNDERGOING IMPACT LOADS
}

\author{
ICARDI $^{*} \mathrm{U}$. and FERRERO ${ }^{* *} \mathrm{~L}$. \\ Dipartimento di Ingegneria Aeronautica e Spaziale \\ Politecnico di Torino - Corso Duca degli Abruzzi 24, 10129 Torino, Italy, \\ fax:+39 (0) 11564 6899, e-mail:
}

ugo.icardi@.polito.it $\quad \underline{\text { laura.ferrero@polito.it }}$

\begin{abstract}
In this study multiple cores sandwich composites undergoing impact loads are optimised in order to improve their strength at the onset of delamination. The optimisation is carried out finding the distribution of the stiffness properties which minimizes the interlaminar stresses. The importance of using refined structural models is emphasised. A 3D Zig-Zag model and a mixed solid element with interlaminar stresses and displacements as nodal d.o.f. are considered as structural models. The former is used within the optimisation process. The latter, which uses a unique set of $\mathrm{C}^{\circ}$ standard tri-linear shape functions for all the d.o.f., i.e. fulfils the intra-element equilibria in an approximate integral form, is used to assess the stress fields of the optimised solution. The onset of delamination is predicted using stressbased criteria. It appears that the optimisation process is able to reduce the interlaminar stress concentrations, with beneficial effects on the delamination resistance for all the sample cases considered.
\end{abstract}

KEY WORDS: Sandwich Structures, Impact Damage Behaviour, Damage Resistance

\footnotetext{
* Associate professor, Dipartimento di Ingegneria Aeronautica e Spaziale, Politecnico di Torino, Turin, Italy

${ }^{* *}$ PhD. student, Dipartimento di Ingegneria Aeronautica e Spaziale, Politecnico di Torino, Turin, Italy
} 


\section{INTRODUCTION}

A peculiar type of sandwich composites is the object of the present paper, the dualcore sandwich composites; they are characterised by one internal face, splitting the core in two parts, having as a results two external faces, one internal one and two cores, as depicted by the Fig. 1 .

Despite it is evident that the impact-induce damage and the damage accumulated in service need structural models which accurately describe the local behaviour, these models are not attractive because they could make the computational effort prohibitively large. The reader is addressed to Noor et al. [1] for a plenty discussion about the models for sandwich composites and to [2-13] for a comprehensive discussion of damage modelling. When sandwich composites are damaged, extremely high, localised stress gradients take place across the thickness (see Icardi [14]).

In the present paper, the time history of the contact force is computed by a $\mathrm{C}^{\circ}$ eightnode plate element based on a 3D zig-zag model, to achieve the best accuracy with the customary five functional d.o.f. This model is also used in the optimization process. A mixed brick element with the three displacements and the three interlaminar stresses as nodal d.o.f. is used to compute the damage at each time step.

At the best of the authors' knowledge no criteria devoted to the analysis of sandwich composites is known which accounts for the local effects at the refined level required by the damage tolerant approach (see, e.g., Soden et al. [6] and Hinton et al. [7]). So, the damage assessment in these materials still remains an open question. Different structural models and criteria will be compared each other in this paper, at the light of previous considerations. The onset of damage is predicted in terms of matrix and fibers failure, cracks, delamination, ripling, wrinkling and face damping using different stress-based criteria, since there is a general agreement that these criteria are accurate enough for this purpose. In this paper the effects of the accumulated damage are accounted for through the ply-discount theory, i.e. using reduced elastic properties for the layers and the cores that failed. For details on this technique see Murray and Schwer [15].

The numerical results presented show the primary role of refined structural models to accurately predict the impact-induced damage of multiple cores sandwiches. They also show the improved damage resistance of these materials and the effectiveness of the optimisation technique, since the incorporation of few optimised plies with the same mean stiffness properties of the plies they substitute can consistently reduce the stresses producing the onset of delamination.

\section{REMARKS ON THE STRUCTURAL MODELS}

In the region where damage rises the interlaminar shear stresses and the transverse normal stress are dominant, while nearby this region the membrane stresses are dominant. If the components are thick (as usual for sandwich composites) the interlaminar stresses are anyway dominant. 


\subsection{Interfacial Contact Conditions}

In order to be accurate, the structural models have to feature continuous interlaminar stresses at the interfaces between the constituent plies and the cores, as required by the elasticity theory. In addition, they should feature a continuous transverse normal stress gradient at these interfaces. In absence of bonding or delamination damage, the displacements components must also be continuous. To contemporaneously fulfill these requirements, the displacements are required to be $\mathrm{C}^{\circ}$ continuous and with suited discontinuous first derivatives at the interfaces. This can be obtained in several ways. In a broad outline, the models can postulate an appropriate piecewise variation of displacements across the laminate thickness, or assume displacements and/or stresses separately for each layer, then impose the interfacial continuity requirements as constraint conditions. The former are the zigzag models. The latter are the discrete-layer models. The mixed brick element used in this paper is a variant of these models. In this element, the displacements and the interlaminar stresses were chosen as nodal d.o.f., with the purpose to automatically fulfil the interfacial conditions on interlaminar stressess and displacements and to capture the extremely high localized stress gradients across the thickness.

The zig-zag model, which is inexpensive and easy to handle, is used in the optimization process, while the mixed solid element, which is accurate but computationally intensive, is used to assess the local effects of the optimized solutions. Hereafter, the basic features of these models will be briefly summarised.

\subsection{Zig-Zag Model}

A third-order zig-zag model with a constant transverse displacement (refer to Icardi [16]) is used to derive the stationary equations for the energy contributions, the higher-order models and particularly the discrete-layer models, being unaffordable for this purpose. In order to improve its accuracy, its strain energy is updated to that of a refined zig-zag model with a high-order piecewise variation of the transverse displacement in the post-processing phase, as described in Icardi [17]. In all the former applications, this model appeared able to provide a realistic prediction of interlaminar stresses, since it correctly models the overall strain energy contributions. Based on this updated model, a eight node, $\mathrm{C}^{\circ}$ plate element was developed by Icardi and Zardo [18] which is used in this paper for computing the contact force time history. Once computed, it is supplied to the mixed solid element and used to compute the impact induced damage.

\subsection{Mixed Solid Element}

When the damage rises, much better results are obtained by the mixed element, developed by Icardi and Atzori [19] assuming the three interlaminar stresses and the three elastic displacements as nodal d.o.f.. Standard $C^{\circ}$, tri-linear serendipity shape functions are used for interpolating the d.o.f.. across the element volume. As a consequence, the intraelement equilibria are met in an approximate integral form.. This choice do not adversely affect the accuracy (see, e.g., Nakazawa [20], and 
Loubignac et al. [21] ). The required level of variation of the layerwise quantities is obtained by increasing the number of subdivisions across the thickness, as customary for discrete-layer models. In this way, the appropriate variation of the quantities of interest in the region where damage rises, is obtained refining the discretisation where it is necessary. Obviously this refinement implies an unavoidable growth of the computational effort. Note that, in all the cases here considered, the processing time and the memory occupation is lower or equal to customary displacement-based counterpart solid elements, while accuracy is improved.

\subsection{Optimization of the Energy Storage}

The optimisation technique recently proposed by the authors (see former applications in Icardi and Ferrero [22-25]) is used in this paper for optimising the energy absorption properties of multi-core sandwiches undergoing impact loads. This technique finds the appropriate in-plane variable distributions of stiffness properties which make extremal certain strain energy contributions of interest. The purpose is to minimise the energy absorbed through unwanted modes (i.e., involving interlaminar strengths) and maximise that absorbed through desired modes (i.e., involving membrane strengths). The stiffness distributions of interest are found solving the Euler-Lagrange equations resulting from assumption of the laminate stiffness properties as the master field and setting to zero the first variation of wanted and unwanted strain energy contributions (e.g., bending, in-plane and outof-plane shears and membrane energies). All the solutions here proposed can be obtained either varying (by currently available manufacturing processes) the orientation of the reinforcement fibres, the fibre volume rate or the constituent materials over the plane of the panels. The effect of this approach is to act as an energy absorption tuning, since it minimises or maximises the amount of energy absorbed by specific modes through a suited in-plane variation of the plate stiffness properties. The first step is to write the first variation of the strain energy for the zigzag model, and use it as a constraint, since it represents the equilibrium. Then the first variation of the strain energy under in-plane variation of the stiffness properties is considered, together with the aforementioned constraint. Setting to zero the first variation of the energy contributions of interest, collecting the contributions that multiply any virtual displacement and applying the Green's theorem, in order the derivatives of the displacements are moved on stiffness terms, the Euler-Lagrange final governing equations are obtained. The number of layers in the laminate and the constituent materials being arbitrary, the extremal problem has sense only in a limited set. In the current case, the optimisation technique is applied to a single ply, finding a stiffness distribution that minimises bending and transverse shears. In the numerical applications, one or few of such optimised layers will be incorporated in the lay-up and the resulting behaviour will be compared to that of the pre-existing solution whose layers have been substituted. This comparison will be carried out always considering optimised layers with the same mean properties of the ones they replace. An approximate solution of the governing equations is used in this paper. This solution of technical interest features a simple parabolic variation of the stiffness properties for each optimised ply. Its coefficients are determined enforcing 
conditions which range from the thermodynamic constraints, to imposition of the mean stiffness, to the choice of a convex or a concave shape, in order to minimise or maximise the energy contributions of interest. Two solutions will be considered. The former reduces the bending of a lamina with moderately increasing the shear stresses, the second one reduces these stresses with a low increment in the bending contribution. These layers will be shown to consistently reduce the interlaminar stresses across the thickness, with beneficial effects on delamination.

\section{MODELLING OF THE CONTACT LOAD}

The low velocity impacts are dominated by delamination and matrix cracking, rather than by penetration-induced fibre breakage, as discussed by Davies and Olsson [26] and Joshi and Sun [27]. Even when the so called Barely Visible Impact Damages (BVID) are not evident, they are always responsible for a relevant strength degradation. There is a general agreement that for this type of impacts strain-rate dependent properties are unnecessary. Thus the material properties can be assumed unchanged with respect to the static case. The contact duration being higher than the stress waves lateral transit time, the plate size and the boundary conditions affect the response, as discussed by Hoo and Fatt [28]. In other words, transverse shear waves reflect off the edges several times while the contact load is still being applied. Hereafter, the appropriate techniques for computing the time history of the contact force will be briefly discussed, since they are basic for the forthcoming assessment of the impact-induced damage.

\subsection{Computation of the Contact Force}

The contact problem can be divided into loading, unloading and reloading phases. In the loading phase, customary modified versions of the Hertzian contact law are used. Various formulas depending on the target and the projectile materials have been proposed. They relate the contact force $F$ with the indentation depth $\alpha$ by a parameter, the contact stiffness $K_{c}$, with assuming various exponents. The exponent $3 / 2$ appears in a very good correlation with a great amount of experimental tests published in literature for carbon, kevlar and glass reinforced composites

$$
F=K_{c} \alpha^{3 / 2}
$$

while $K_{c}$ needs to be estimated for each case. To this purpose, various formulas depending on the target and the projectile materials have been proposed. In place of experiments, non-linear interface finite elements can be conveniently used to determine the relation between force and the indentation depth. Several commercial computers codes with the capability to accurately simulate the contact problem are to date available. In the present paper, MECALOG-RADIOSS was used for this purpose. Once the contact force has reached its maximum and the relative motion between the target structure and the projectile changes its sign, the loading phase ends. In this unloading phase, a permanent indentation occurs even when the 
laminates are thin, thus a new law have to be used. In the present paper this part of the time history is those described by Crook [29]:

$F=F_{m}\left(\frac{\alpha-\alpha_{0}}{\alpha_{m}-\alpha_{0}}\right)^{q}$

in which $F_{m}$ is the load at which the unload phase starts (i.e., the maximum contact force), $\alpha_{m}$ is the relative indentation depth, while $\alpha_{0}$ is the permanent indentation depth. While the paramenters $F_{m}$ and $\alpha_{m}$ are easily determined from the loading phase curve, $\alpha_{0}$ have to be estimated. In the present paper this was carried out by a finite element analysis, using RADIOSS and a simple model made by brick and shell elements. According to the experiments presented in literature, the exponent $q$ was set to 2.5. This unloading law was used every time the contact force decreased. In the event of bounces also a reloading law is required. The indentation in this phase has to be the difference among the permanent and the one currently occurring $\alpha$ $\alpha_{0}$. The following reloading law is used in this paper:

$$
F=k_{c}{ }^{\prime}\left(\alpha-\alpha_{0}\right)^{p}
$$

Because in agreement with the experiments, the exponent $p$ was set to 1.5. This approach for modelling the contact force was chosen because it appeared accurate in all the papers in which a comparison was made with experiments and, furthermore, because it is easy to be implemented and requires a low computational effort.

\subsection{Solution of Dynamic Equations}

The impact force computed as outlined above is applied at the impact point. The projectile is schematised as a point mass, since the $3 \mathrm{D}$ analysis by RADIOSS evidenced a negligible deformation. The force acting on it is assumed equal to the contact force if $\geq 0$, or to vanish if $<0$. Both the $\mathrm{C}^{\circ}$ eight node plate element based on the zig-zag model presented by Loubignac et al. [21] and the method of weighted residuals in the Galerkin's version are used as structural models in the computation of the contact force, because modelling errors are deleterious in this phase, as selfevident. The plate element having been described into details in previous papers, only a basic remark on the closed form model is reported hereafter. It minimizes the errors by ortogonalising with respect to the set of trial functions, which are required to closely represent the deformation modes over the whole domain and to fulfil the geometrical boundary conditions, as explained by Timoshenko [30]. Note that a large number of components (i.e., up to 15) is required to adequately represent the waves which transit and reflect at the edges. For this reason, the meshing have to be adequately refined when the analysis is carried out by the eight-node plate element. The two discretisation, e.g. by the Galerkin's method and by the finite element, give rise to dynamic equations which are solved using the Newmark's algorithm, as customary. An iterative approach is required, since the magnitude of 
the contact force is expressed as a nonlinear relation of displacements and the time step must be very small. Once convergence is reached, the displacements, velocities and accelerations vectors are computed at any time step and with this information the strains and the stresses at each point of the laminate are determined.

\subsection{Assessment of the Contact Force modelling}

The accuracy of the contact force model outlined above was assessed comparing present predictions by the plate element with updated strain energy to the experimental results of the sample case dealt with by Takle [31]. Only this case is here discussed, because the procedure has already been successfully compared with a wide number of experimental results published in literature, in terms of penetration time history, structure and projectile motion and kinetic energy (e.g. Choi [32], Prasad [33]) and with several experimental tests made by the authors and object of a paper actually in publication.

It consists of a Divinicell H80 polymer foam core sandwich panel for marine applications with multiaxial GRP laminated faces, which is subjected to a drop weight impact test. The test rig consists of a quadratic frame with light opening of $570 \times 570 \mathrm{~mm}$ which supports the sandwich plate through steel pipes with diameter $40 \mathrm{~mm}$, welded together at the corners (for details about the test rig, refer to Tackle [31], where the experimental results are published). A steel projectile with a mass of $6.2 \mathrm{~kg}$ and a diameter of $75 \mathrm{~mm}$, which mounts an accelerometer on its top, is let fall down from a height of $3.5 \mathrm{~m}$, giving an impact energy of $213 \mathrm{~J}$. Length and width of the panel are $620 \mathrm{~mm}$, while the overall thickness is $31 \mathrm{~mm}$, the thickness of the face layers being $3 \mathrm{~mm}$ and that of the core being $25 \mathrm{~mm}$. The lay-up of the sandwich panel is: $\left[0^{\circ} / 90^{\circ} / 90^{\circ} / 0^{\circ}\right.$-core $\left.-0^{\circ} / 90^{\circ} / 90^{\circ} / 0^{\circ}\right]$. The properties of the face layers are the following ones: Young's moduli $E_{1}=E_{2}=31.5 \mathrm{Gpa}, E_{3}=8.2 \mathrm{Gpa}$, Poisson's ratios $v_{12}=v_{23}=v_{13}=0.2$, shear moduli $G_{12}=6.0 \mathrm{Gpa}, \mathrm{G}_{13}=\mathrm{G}_{23}=5.1436 \mathrm{Gpa}$, density $1.97 \mathrm{~g} / \mathrm{cm}^{3}$. The strengths are: $\mathrm{S}_{\mathrm{x}}=\mathrm{S}_{\mathrm{y}}=482 \mathrm{Mpa}, \mathrm{S}_{\mathrm{z}}=65 \mathrm{Mpa}, \mathrm{S}_{\mathrm{xy}}=55.9 \mathrm{Mpa}$, $\mathrm{S}_{\mathrm{xz}}=\mathrm{S}_{\mathrm{yz}}=30 \mathrm{Mpa}$.

The properties of the foam core, which is assumed to be isotropic, are: Young's modulus $E=85 \mathrm{Mpa}$ in compression and $80 \mathrm{Mpa}$ in tension, shear modulus $\mathrm{G}=31$ Mpa, Poisson's ratiov $=0.32$, density $0.08 \mathrm{~g} / \mathrm{cm}^{3}$; strengths: $1.2 \mathrm{Mpa}$ in compression and 2.2 Mpa in tension. The properties of the projectile and of the frame are: bulk modulus 401,3 Gpa, shear modulus $87 \mathrm{Gpa}$, density $7.5 \mathrm{~g} / \mathrm{cm}^{3}$.

The comparison between the results by the plate element with updated strain energy and the experiment made by Takle [31] are reported in Fig.2. Results in a close agreement have been obtained using the Zig-Zag model and the Galerkin's closed form approach, which are omitted to contain the length of the paper. The following observations could be made. It appears that accounting for the properties degradation, a much accurate representation of the time-force is obtained in the unloading phase (i.e. after the peak), while, obviously, no significant improvement is found before. The numerical tests carried out for this case show that in the cases in which the time history develops oscillations of physical nature, the quality of predictions can be consistently improved using appropriate loading, unloading and reloading laws like those adopted in the present paper. In addition, it is seen the 
beneficial effect on accuracy of modelling the transverse shear with a sophisticated model, since much accurate results are obtained with respect to conventional models, as shown in Fig.2; in fact using a refined zig-zag model for describing the displacement field leads to a contact force prediction very close to the one obtained experimentally; on the contrary, choosing low-order equivalent single layer models (among others the wide spread First Order Shear Deformation Plate Theory, FSDPT and Classical Laminated Plate Theory, CLT); in Fig.2 a comparison with these two low-order models is also presented. The good agreement among predictions and experiments allows us to assume as appropriate the modelling of the contact force of Section 3.3.1.

\subsection{Damage Criteria}

Fibers and matrix failure is predicted using the Hashin's criterion, developed for unidirectional composites; in-situ strengths are used to account for the interaction between the plies, since there can be a remarkable difference between the strength of a ply when isolated and when embedded in a laminate. In this way, the information available for unidirectional composites can be used for laminates. The onset of delamination is predicted using the Choi-Chang (see Eq. (4)), Chai-Gädke (see Eq. (5)), and an heuristic criterion proposed by Icardi [17] (see Eq. (6)). These criteria were chosen because they use only standard, easy to assess, engineering ply properties, they do not require curve-fitting parameters that have to be determine by experiments, and appeared accurate in the applications presented in literature.

A brief description of these criteria will be presented; delamination between nth layer and $(\mathrm{n}+1)$ th layer occurs when the failure index $e_{d}{ }^{2}>1$; in the Choi-Chang's criterion, the failure index has the following formulation:

$D_{a}\left[\left(\frac{{ }^{n+1} \bar{\sigma}_{22}}{{ }^{n+1} Y^{t}}\right)^{2}+\left(\frac{{ }^{n+1} \bar{\sigma}_{13}}{{ }^{n+1} S^{i s}}\right)^{2}+\left(\frac{{ }^{n} \bar{\sigma}_{23}}{{ }^{n} S^{i s}{ }_{23}}\right)^{2}\right]=e_{d}{ }^{2}>1$

where $\bar{\sigma}_{i j}$ are the stress mean value across the thickness of the individual ply, $D_{a}$ is an empirical constant depending on the material properties, $S^{\text {is }}$ and $Y^{t}$ are respectively the in-situ shear and tensile strengths, computed by the Chang and Lessard formula (see Chang and Lessard [12]). In the Chai-Gädke's criterion, the failure index is computed according to the following formula:

$f_{1}\left(\frac{{ }^{n} \bar{\sigma}_{22}}{{ }^{n} Y^{t}}\right)^{2}+f_{2}\left(\frac{{ }^{n+1} \bar{\sigma}_{23}}{{ }^{n+1} S^{i s}{ }_{23}}\right)^{2}+f_{3}\left(\frac{{ }^{n} \bar{\sigma}_{13}}{{ }^{n} S^{i s}{ }_{13}}\right)^{2}=e_{d}{ }^{2}$

where: $f_{1}=\frac{{ }^{n} t}{{ }^{n+1} t}\left(\frac{{ }^{n} Q_{11}-{ }^{n+1} Q_{11}^{\prime}}{{ }^{n} Q_{11}-{ }^{n+1} Q_{22}}\right) ; f_{2}=0.5\left(1+\frac{{ }^{n+1} G_{23}}{{ }^{n} G_{23}} \cos ^{2} \Delta \theta+\frac{{ }^{n+1} G_{23}}{{ }^{n} G_{23}} \sin ^{2} \Delta \theta\right)$; 
$f_{3}=0.94\left(1+\frac{{ }^{n+1} G_{13}}{{ }^{n} G_{13}} \cos ^{2} \Delta \theta+\frac{{ }^{n+1} G_{13}}{{ }^{n} G_{13}} \sin ^{2} \Delta \theta\right) ;$ being ${ }^{n+1} Q_{11}^{\prime}$ the transformed stiffness of the $(n+1)$ th ply in the fiber direction, as described by Chai and Gädke [34]. Finally, the euristic criterion proposed by Icardi [17] is made up of two statements, in order to take into account the effect of the transverse tensile stress, effect which is beneficial when the stress is a compression, detrimental otherwise:

$\pm\left(\frac{\bar{\sigma}_{33}}{{ }^{t / c} S^{i s}{ }_{33}}\right)^{2}+\left(\frac{\bar{\sigma}_{23}}{S^{i s}{ }_{23}}\right)^{2}+\left(\frac{\bar{\sigma}_{13}}{S^{i s}{ }_{13}}\right)^{2}+\left(\frac{\bar{\sigma}_{22}}{S^{i s}{ }_{22}}\right)^{2}=e_{d}^{2}$

with $\left[+;{ }^{t} S^{i s}{ }_{33}\right]$ for $\bar{\sigma}_{33} \geq 0$ and $\left[-;{ }^{i} S_{33}\right]$ for $\bar{\sigma}_{33}<0$.

The degradation of the properties after fibres and matrix failures or delamination is simulated using the ply-discount theory (see Murray and Schwer [15]), i.e. by locally reducing the stiffness of the failed plies in the on-axis constitutive equation. The elastic moduli are degraded according to the following rule:

$Q_{\text {after }}=Q_{\text {before }} 10^{-n}$ where $0 \leq n \leq 20$

$Q_{\text {after }}$ and $Q_{\text {before }}$ being Young's and shear elastic moduli selected according to the type of failure occurred. Assuming $n=0$, we have no degradation, while, e.g., assuming $n=1$, we have a degradation of the $10 \%$. In case of fibres failure, $E_{11}, G_{12}$, and $v_{12}$ are reduced according to this rule, while in the case of matrix failure $\mathrm{E}_{22}$, $\mathrm{G}_{12}$, and $v_{12}$ are reduced. When delamination starts, $\mathrm{G}_{13}$ and $\mathrm{G}_{23}$ are reduced. The suited value of the exponent $n$ have to be chosen from the values suggested in literature, or varying it till the results fit available experimental observations.

\section{NUMERICAL RESULTS}

Numerical applications are presented hereafter for multiple-core sandwich composites. First, the characteristic stress fields in these materials are assessed, subsequently their impact behaviour and the improvements brought by the present optimisation procedure are discussed. An interesting favourable property of these materials is their improved damage resistance, since the presence of multiple cores and faces inhibits the deleterious spreading of failure or damage of classical sandwich composites. Although the intermediate faces does not contribute to the bending stiffness like the external ones, the multiple-core sandwich composites do not render vain the advantages in terms of weight offered by sandwich composites, if the damage tolerance is considered. This is just because the classical sandwiches need to be over-sized in order to tolerate the damage growth in service. The improved damage resistance of multiple-core sandwich composites is shown in Fig.3 (undamaged case) and Fig.4, which depict the interlaminar shear stress across the thickness of a simply-supported plate in cylindrical bending under a sinusoidal distributed loading. In these figures the results obtained by the mixed solid element are compared to the exact elasticity solution presented by Icardi [17], in order to further assess the accuracy of this element, already successfully 
assessed in the former applications. With exact elasticity solution the authors refer to a closed form approach, that, due to its simple loading and boundary conditions, has an exact solution to the three-dimensional Elasticity equations, see Pagano [35] and [36]. The better residual properties of multiple core materials appear through their capability to bear stresses also when an interface is damaged or one of the cores failed, as appearing in Fig.4, while the classical sandwiches cannot. The material properties for the sample cases of Fig. 3 and Fig. 4 are summarized in the Table 1.

The results refer to a length-to-thickness ratio of 4 , in order to make severe the test for the numerical models. The materials [1] to [4] of Table 1 are stacked with the following sequence $([1] /[2] /[3] /[1] /[3] /[4]) s ;$ The thickness ratio of the constituent layers with respect to the total thickness is: $(0.01 / 0.025 / 0.015 / 0.02 / 0.03 / 0.4)_{s}$. The results are reported in normalized form, i.e. $\sigma_{\mathrm{xz}}{ }^{*}=\sigma_{\mathrm{xz}} / \mathrm{P}^{\circ}, \mathrm{P}^{\circ}$ being the magnitude of the sinusoidal loading. The capabilty of multiple-core sandwich composites to undergo loads when damaged is evidenced by the comparison with the results of the classical sandwich case reported in the top right corner. The comparison with the elasticity solutions of Fig.3 having shown the accuracy of the solid element also in the current case of multiple-core sandwich composites, the impact behaviour of these material can now be investigated.

To this purpose, let us consider a sandwich beam with two cores, two external faces and an internal one, $20 \mathrm{~cm}$ long, and $2 \mathrm{~cm}$ thick, with a width of $1 \mathrm{~cm}$. The material for the faces is indicated by $[\mathrm{F}]$ in Table 2, while that of the cores is indicated as $[\mathrm{C}]$. The stacking sequence is $[0 / 90 / 0 / \mathrm{c} / 0]_{\mathrm{s}}$, where $\mathrm{c}$ indicates the core. The plies constiting the layers and the core are assumed to have a thickness of $\left[0.01_{3} / 3 / 0.01\right]_{\mathrm{s}}$, respectively.

The sandwich beam is simply-supported at the edges and subjected to an impact load in the middle. An optimization with the technique outlined in Sect. 2.4 has been performed in order to find a face layer with a variable stiffness distribution, able to reduce the strong interlaminar shear stress concentration nearby the core interface responsible for the damage formation and growth. This layer is assumed to have the same mean stiffness of the layer it replaces. Further conditions derive from the imposition of thermodynamics constraints. It is here reminded that the optimal stiffness coefficients distribution is determined by the present optimization technique making extremal the energy contributions of interest. In the current case, they are determined by minimising the interlaminar stresses and maximising the bending energy. The coefficients appearing in the expressions of the plate stiffness, which are variable with the in-plane coordinates, are fixed by the conditions above mentioned about the mean value and thermodynamics considerations. The law of variation for the stiffness coefficients assumed in this paper is represented in Fig.5, in function of their mean value. This choice is motivated by the need for finding a solution of technical interest which is compatible with current manufacturing technologies. It represents a sub-optimal, second-order polynomial approximation for the transformed reduced stiffness coefficients which makes extremal the energy contributions in each constituent ply. It provides an exact solution to the conditions involving second order derivatives of the stiffness coefficients and an approximate solution for higher-order ones. Note that many of these conditions identically vanish when the optimisation is carried out for a single ply, as in the present study (see Icardi and Ferrero [22-25]). In order to reduce the interlaminar shear stresses 
responsible for the delamination damage after a low velocity impact, the coefficients of the chosen second-order polynomial approximation were set in order to carry out an energy transfer from the shear mode to the in-plane one, with respect to the preexisting lamina with a constant stiffness distribution.

This solution, which produces a slight increment in the bending contribution and in the transverse normal stress, is able to consistently reduce the transverse shear stress concentration induced by the impact at the interface between the outer face layer and the cores, as shown in Fig.6, even when substituting a single optimised layer with the same mean stiffness properties of the layer it replaces. Fig.7 shows the slight increment in the transverse normal stress with respect the classical layer. The substituted layers are the one oriented at $0^{\circ}$ in the outer faces in the beam with a constant distribution of stiffness properties.

Let now consider the same sandwich beam before mentioned, but having a fixed edges and being loaded by an impact force at the free edge. The beneficial effects of the optimised layer incorporation on the strength at the onset of delamination are shown by Fig. 8 to Fig.10, which depict the reduction of the failure index across the thickness produced by the incorporation of a layer with optimised properties, respectively according to the Choi-Chang, Chai-Gädke, and an heuristic criterion described by lcardi [17], whose statements are reported in subsection 3.4, equations (4), (5) and (6).

The advantages of the optimization technique based on a variation of the stiffness properties over the plane of the plies here proposed appears to be effective by previous Fig. 8 to Fig.10. The present application relies with sandwich structures in multiple-core configuration, but previous applications to laminated composites (refer to Icardi and Ferrero [22-25]) evidenced the same kind of advantages. It is worthwhile to note that this optimisation technique can be applied to all the cases based on energy formulations, i.e. it is applicable to various engineering cases where a critical behaviour may be expressed in terms of its energy absorption modes.

\section{CONCLUSIONS}

In this paper the behaviour of multiple core sandwich composites, undergoing impact loads, and their optimisation were studied. A mixed solid element with interlaminar stresses and displacements as nodal d.o.f. was used in order to predict the interlaminar stresses with accuracy. Characteristic feature, all the nodal d.o.f. are discretised by a unique set of standard $\mathrm{C}^{\circ}$, tri-linear shape functions, so the intra-element equilibria are met in an approximate integral form. The optimisation is performed by finding the distribution of the laminate stiffness coefficients making extremal the energy contributions of interest, i.e. minimum the transverse shear energy and maximum the membrane energy. The structural model for the optimisation is a zig-zag model, in order to have a precise descriptions of energy contributions with a low mathematical effort. It appears that the delamination resistance of multiple-core sandwich materials, which is already better than their classical counterparts, can be improved incorporating a variable stiffness ply. It also appears the importance of modelling the impacts in these materials using sophisticated models. 


\section{REFERENCES}

[1] Noor, A.K., Burton, W.S., Bert, C.W., "Computational models for sandwich panels and shells", Applied Mechanical Reviews, No.49, pp 155-199, (1996).

[2] Nahas, M.N., "Survey of Failure and Post-Failure Theories of Laminated Fibre Reinforced Composites", J. Compos. Technol. Res., No.8, pp 138-153, (1986).

[3] Paris, F., "A Study of Failure Criteria of Fibrous Composite Materials", NASA/CR-2001-210661, (2001).

[4] Tennyson, R.C. and Wharam, G.E., "Evaluation of Failure Criterion for GraphiteEpoxy”, NASA CR-172547, (1985).

[5] Tong, L. , "An Assessment of Failure Criteria to Predict the Strength of Adhesively Bonded Composite Double Lap Joints", J. Reinf. Plast. Compos., No.16 (8), pp 698-713, (1997).

[6] Soden, P.D., Hinton, M.J., Kaddour, A.S., "Biaxial Test Results for Strength and Deformation of a Range of e-Glass and Carbon Fibre Reinforced Composite Laminates: Failure Exercise Benchmark Data", Compos. Sci. Technol., No.62, pp 1489-1514, (2002).

[7] Hinton, M.J., Kaddour, A.S., Soden, P.D., "A Comparison of the Predictive Capabilities of Current Failure Theories for Composite Laminates, Judged Against Experimental Evidence", Compos. Sci. Technol., No.62, pp 1725-1797, (2002).

[8] Rowlands, R.E., "Strength (Failure) Theories and Their Experimental Correlations", Handbook of Composites 3, G.C. Sih, and A.M. Skudra, eds, Elsevier New York, pp 71-125, (1985).

[9] Choi, H.Y.T., Wu, H.Y.T., Chang, F.K., "A New Approach Toward Understanding Damage Mechanism and Mechanics of Laminated Composites Due to LowVelocity Impact: Part II-Analysis", J. Compos. Mater., No.25, pp 1012-1038, (1991).

[10] Choi, H.Y. and Chang, F.K., "A Model for Predicting Damage in Graphite/Epoxy Laminated Composites Resulting From Low-Velocity Point Impact”, J. Compos. Mater., No.26, pp 2134-2169, (1992).

[11] Lee, Y.J. and Huang, C.H., "Ultimate Strength and Failure Process of Composite Laminated Plates Subjected to Low-velocity Impact", J. Reinf. Plast. and Compos., No.10, pp 542-556, (1991).

[12] Chang, F.K. and Lessard, L., "Damage tolerance of laminated composites containing an open hole and subjected to compressive loadings: Part IAnalysis", J. Compos. Mater., No.25, pp 2-43, (1990).

[13] Wang, C.Y. and Yew, C.H., "Impact Damage in Composite Laminates", Compos. Struct., No.37, pp 967-982, (1990).

[14] Icardi, U., "Applications of Zig-Zag Theories to Sandwich Beams", Mechanics of Advanced Materials and Structures, No.10 (1), pp 77-97, (2002).

[15] Murray, Y. and Schwer, L., "Implementation and Verification of FiberComposite Damage Models", Failure Criteria and Analysis in Dynamic Response, ASME AMD, No.107, pp 21-30, (1990).

[16] Icardi, U., "Higher-order Zig-Zag Model for Analysis of Thick Composite Beams With Including Transverse Normal Stress and Sublaminates 
Approximations", Composites-Part B: Engineering, No.32b, pp 343-354, (2001).

[17] Icardi, U., " $C^{\circ}$ Plate Element Based on Strain Energy Updating and Spline Interpolation, for Analysis of Impact Damage in Laminated Composites", Int. J. Impact Eng., In press.

[18] Icardi, U. and Zardo, G., " $\mathrm{C}^{\circ}$ plate element for delamination damage analysis based on a zig/zag model and strain energy updating", Int. J. Impact Eng., No.31(5), pp 579-606, (2005).

[19] Icardi, U. and Atzori, A., "Simple, Efficient Mixed Solid Element for Accurate Analysis of Local Effects in Laminated and Sandwich Composites", Adv. Eng. Software, No.35, pp 843-859, (2004).

[20] Nakazawa, S., "Mixed Finite Elements and Iterative Solution Procedures", Iterative methods in non-linear problems, Pineridge, (1984).

[21] Loubignac, C., Cantin, C., Touzot, C., "Continuous Stress Fields in Finite Element Analysis", AIAA J, No.15, pp 1645-1647, (1978).

[22] Icardi, U. and Ferrero, L., "A study of energy absorption in fiber-reinforced composites: transfer from bending to shear", Proceedings of the $3^{\text {rd }}$ International Conference on Structural Stability and Dynamics, June 19-22nd, Kisimmee, Florida, USA, (2005).

[23] Icardi, U. and Ferrero, L., "Impact and Blast Pulse: Improving energy absorption of fibre-reinforced composites through optimized tailoring", Proceedings of the $8^{\text {th }}$ Biennial ASME Conference on Engineering System Design and Analysis, Paper ESDA2006-95772, July 4-7th, Turin, Italy, (2006).

[24] Icardi, U. and Ferrero, L., "Improving energy absorption and dissipation of composites through optimized tailoring", Proceedings of the IMECE2006ASME International Mechanical Engineering Congress and Exposition, Paper IMECE2006-13349, November 5-10th, Chicago, Illinois, USA, (2006).

[25] Icardi, U. and Ferrero, L., "Optimized tailoring for reducing interlaminar stress accumulation in fibre-reinforced composites", Proceedings of the ICCMS-06, $2^{\text {nd }}$ International Congress on Computational Mechanics and Simulation, December 8-10 ${ }^{\text {th }}$, IIT Guwahati, India, (2006).

[26] Davies, G.A. and Olsson, R., "Impact on Composite Structures", The Aeronautical Journal, No.11, pp 541-564, (2004).

[27] Joshi, S.P. and Sun, C.T., "Impact-induced Fracture Initiation and Detailed Dynamic Stress Field in the Vicinity of Impact", Proc. American Society of Composites $2^{\text {nd }}$ Tech. Conf., September 23-25th, DE, pp 177-185, (1987).

[28] Hoo, L.C. and Fatt, M.S., "Perforation of Composite Plates and Sandwich Panels", J. Compos. Mater., No.40 (20), pp 1801-1839, (2006).

[29] Crook, A.W., "A Study of Some Impacts Between Metal Bodies by a Piezoelectric Method", Proc. of the Royal Society, London, Series A, No.212, p. 377, (1952).

[30] Timoshenko, S.P. and Woinosky-Krieger, S., "Theory of Plates and Shells", $2^{\text {nd }}$ edition, McGraw-Hill, (1969).

[31] Takle, S., "Experimental and Numerical Studies of Impact Behaviour of GRP reinforced Composite Sandwich Materials", University of Oslo, Research Report In Mechanics No.5, ISBN 82-553-1417-2, ISSN 0801-9940, (2003). 
[32] Choi, H.I., "Contact force history analysis of composite sandwich plates subjected to low-velocity impact", Composite Structures, No.75, pp 582-586, (2006).

[33] Prasad, C.B., Ambur, D.R., Starnes, J.H, "Response of Laminated Composite Plates to Low-speed Impact by Different Impactors", AIAA Journal, No.32 (6), pp 1270-1277, (1994).

[34] Chai, Y. and Gädke, M., "Impact damage simulation and compression after impact of composite stiffened panels", DLR Report 1B, No.20, pp 131-199, (1999).

[35] Pagano, N.J., "Exact solutions for composite laminates in cylindrical bending", Journal of Composite Materials, No.3, pp 398-411, (1969).

[36] Pagano, N.J., "Exact solutions for rectangular bidirectional composites and sandwich plates", Technical paper, USAF Materials Lab Wright-Patterson AFB, Ohio, (1970).

Table 1. Material properties for the sandwich panels analyzed in Fig. 3 and Fig.4.

\begin{tabular}{|c|c|c|c|c|}
\hline Material & $\mathrm{E}_{11}[\mathrm{Gpa}]$ & $\mathrm{E}_{13}[\mathrm{Gpa}]$ & $\mathrm{G}_{13}[\mathrm{Gpa}]$ & $v_{13}$ \\
\hline$[1]$ & 1 & 1 & 0.2 & 0.25 \\
\hline$[2]$ & 33 & 33 & 8 & 0.25 \\
\hline$[3]$ & 25 & 25 & 0.5 & 0.25 \\
\hline$[4]$ & 0.05 & 0.05 & 0.0217 & 0.15 \\
\hline
\end{tabular}

Table 2. Material properties for the sandwich beam analyzed in Section 4, Numerical Results

\begin{tabular}{|c|c|c|c|c|c|c|}
\hline Material & $\mathrm{E}_{11}[\mathrm{Gpa}]$ & $\mathrm{E}_{13}[\mathrm{Gpa}]$ & $\begin{array}{c}\mathrm{G}_{13} \\
{[\mathrm{Gpa}]}\end{array}$ & $\begin{array}{c}\mathrm{G}_{23} \\
{[\mathrm{Gpa}]}\end{array}$ & $v_{13}$ & $v_{23}$ \\
\hline$[\mathrm{F}]$ & 142 & 9.8 & 7.1 & 3.3 & 0.3 & 0.5 \\
\hline$[\mathrm{C}]$ & 0.138 & 0.138 & 0.41 & 0.024 & 0.02 & 0.02 \\
\hline
\end{tabular}




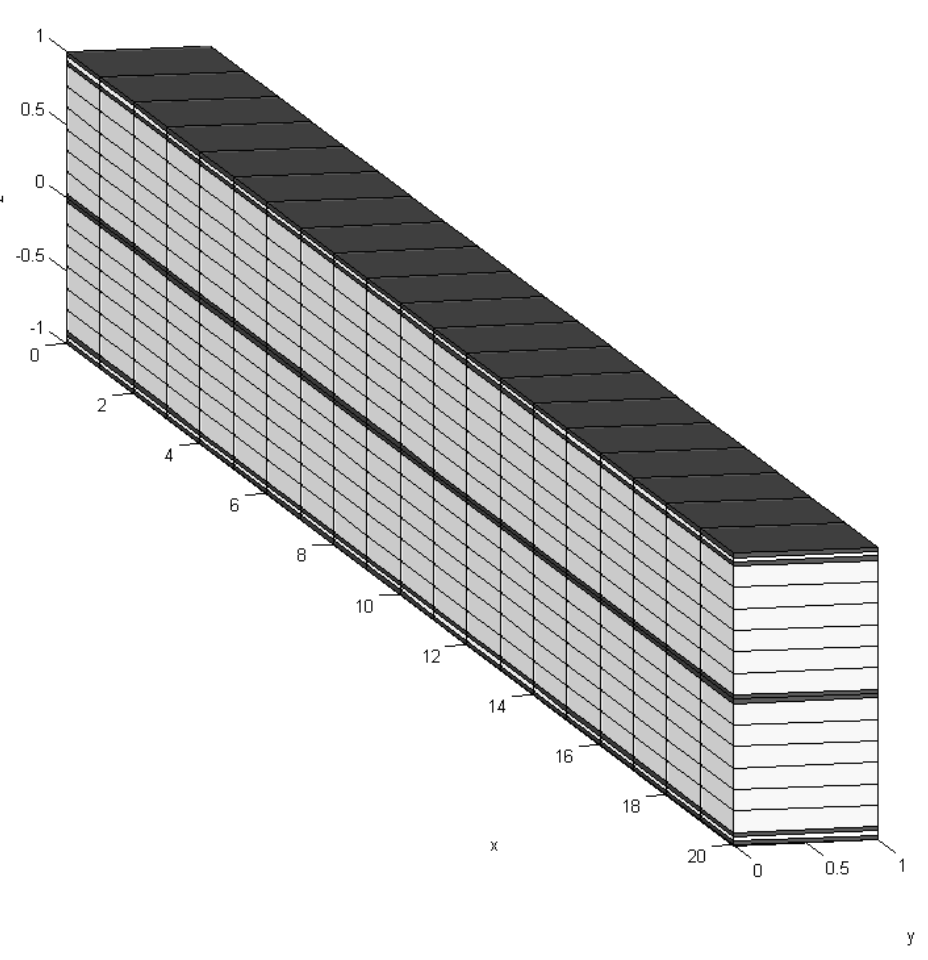

Fig.1. Example of dual core sandwich beam, two outer faces, an inner face and two cores.

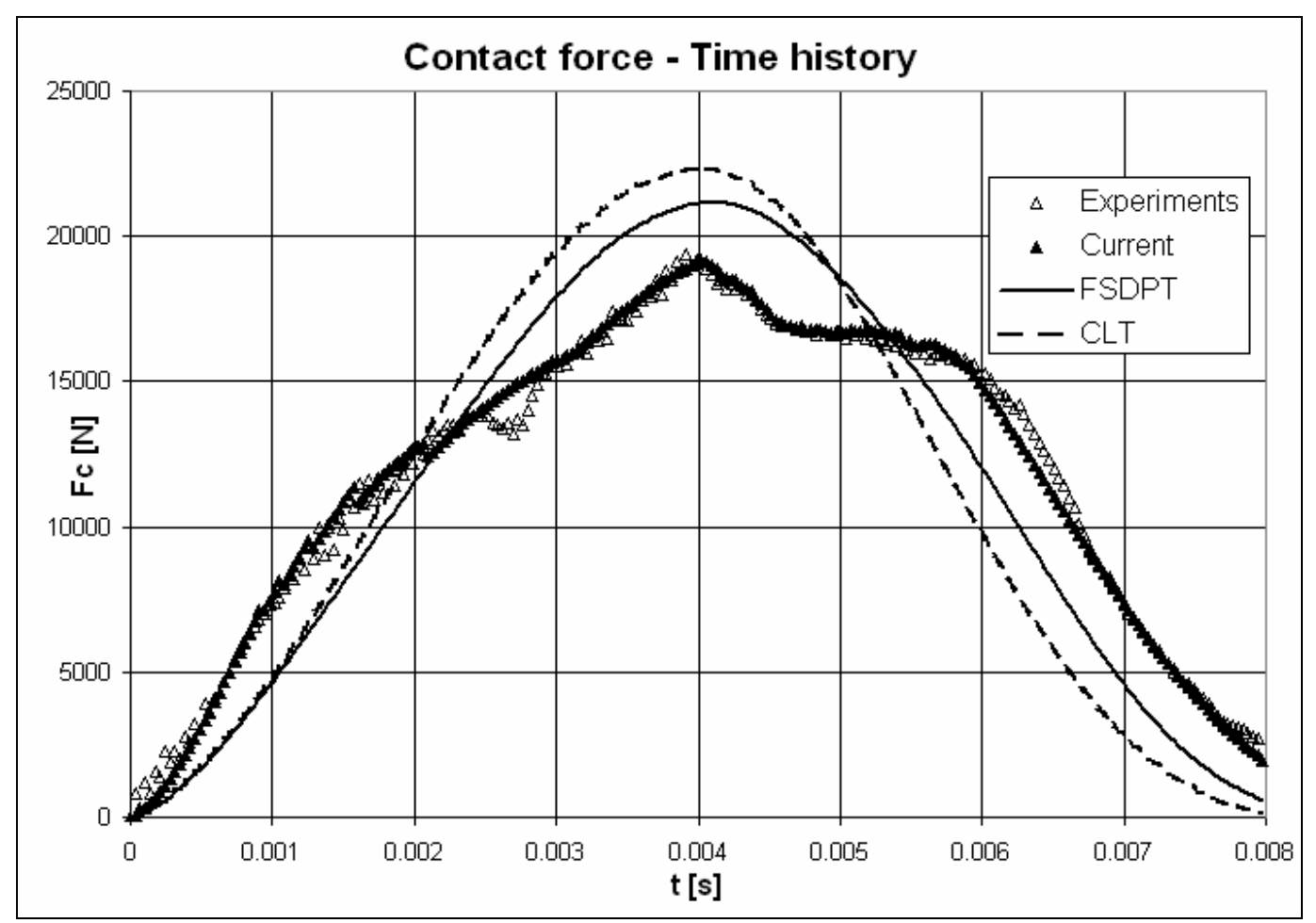

Fig.2. Experimental and numerical contact force time history of a sandwich panel. 


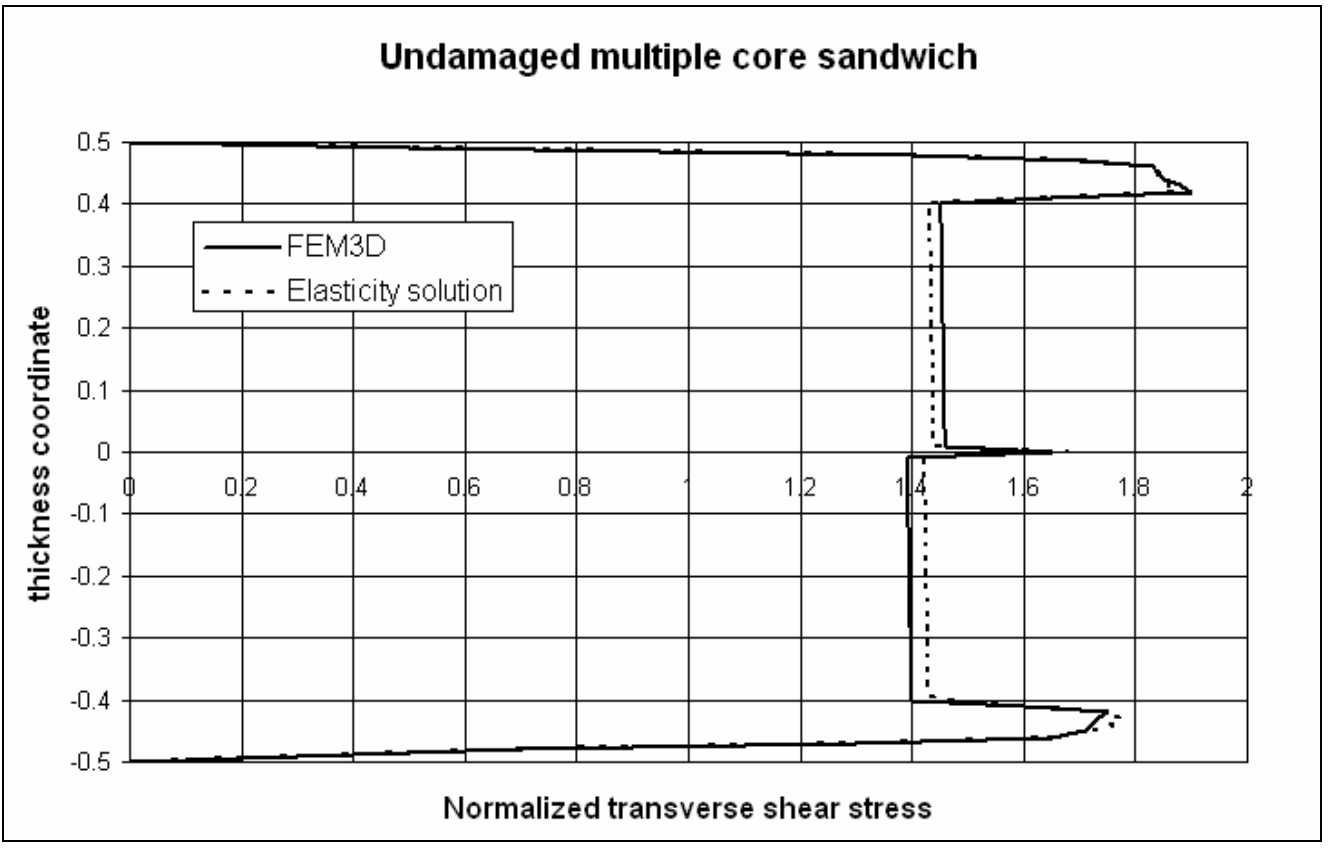

Fig.3. Normalized transverse shear stress across the thickness for an undamaged multiple core sandwich, comparison between elasticity and numerical solution (3D mixed element).

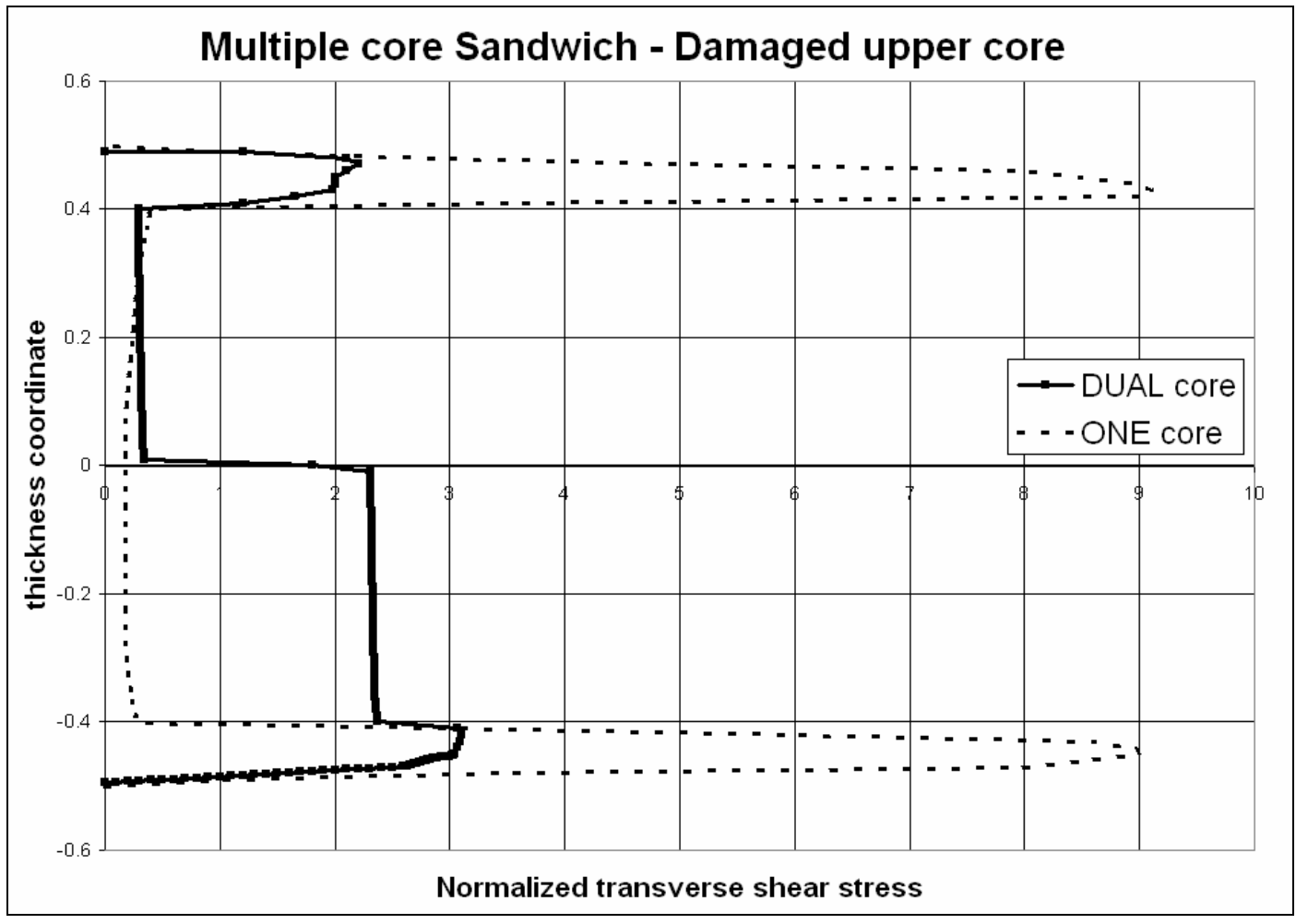

Fig.4. Normalized transverse shear stress across the thickness for a damaged sandwich, comparison between one core and dual core sandwich (FEM results). 


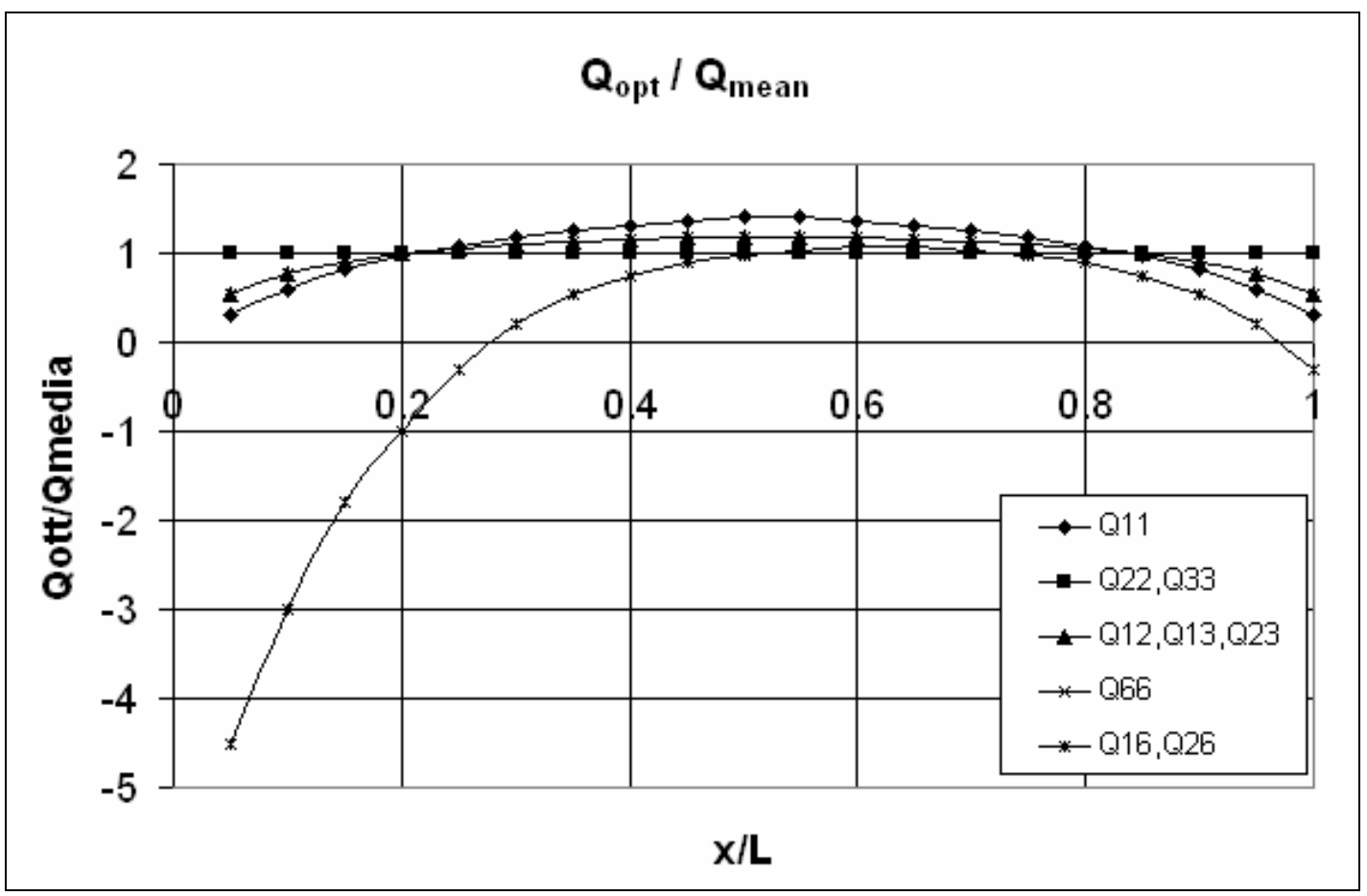

Fig.5. Stiffness contributions variation in the spanwise direction for the optimized layers, transfer from shear to membrane energy.

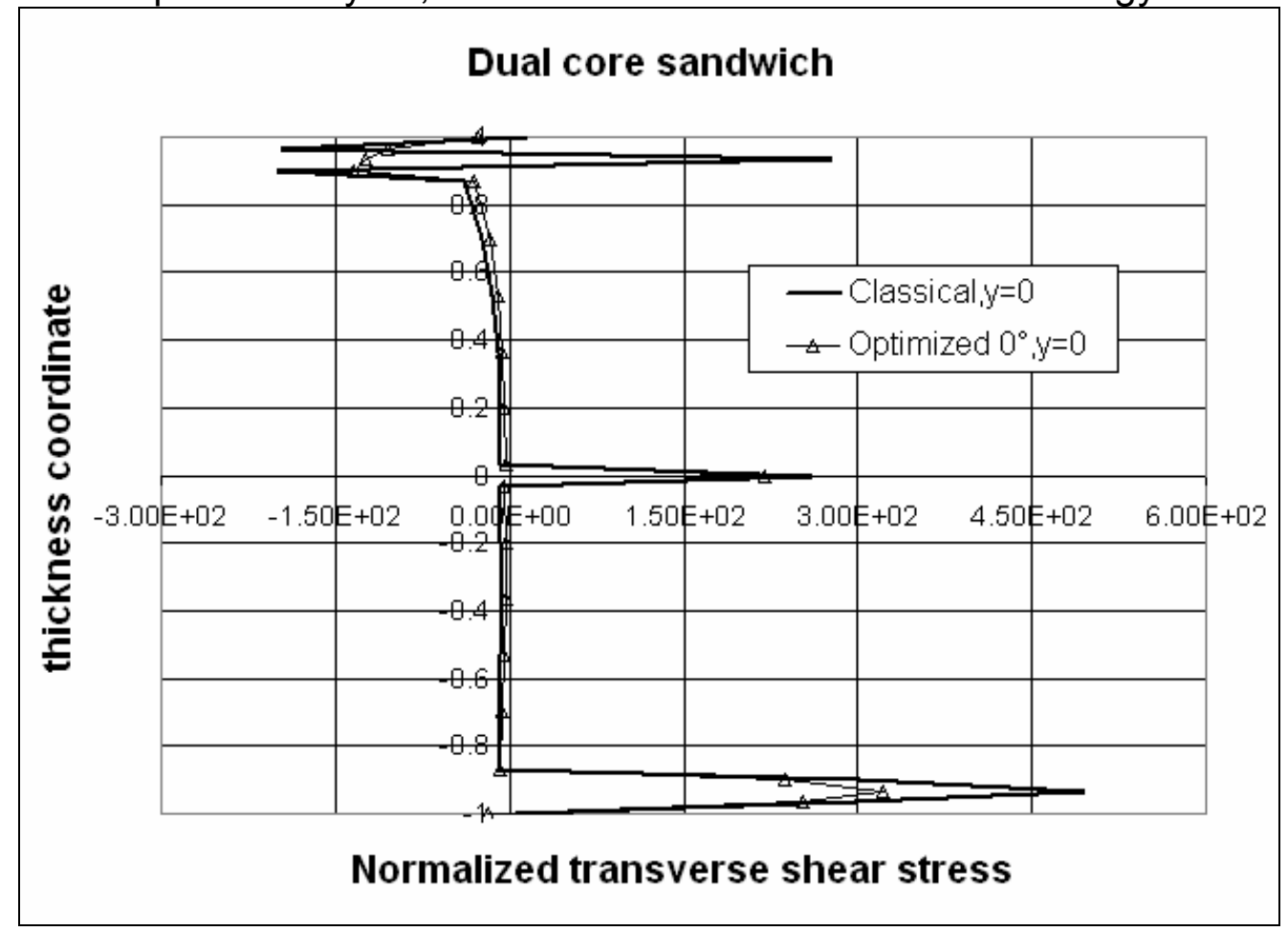

Fig.6. Through-the-thickness normalized transverse shear stress, at the impact point. Comparison between constant stiffness plies (Classical) and variable one (Optimized). 


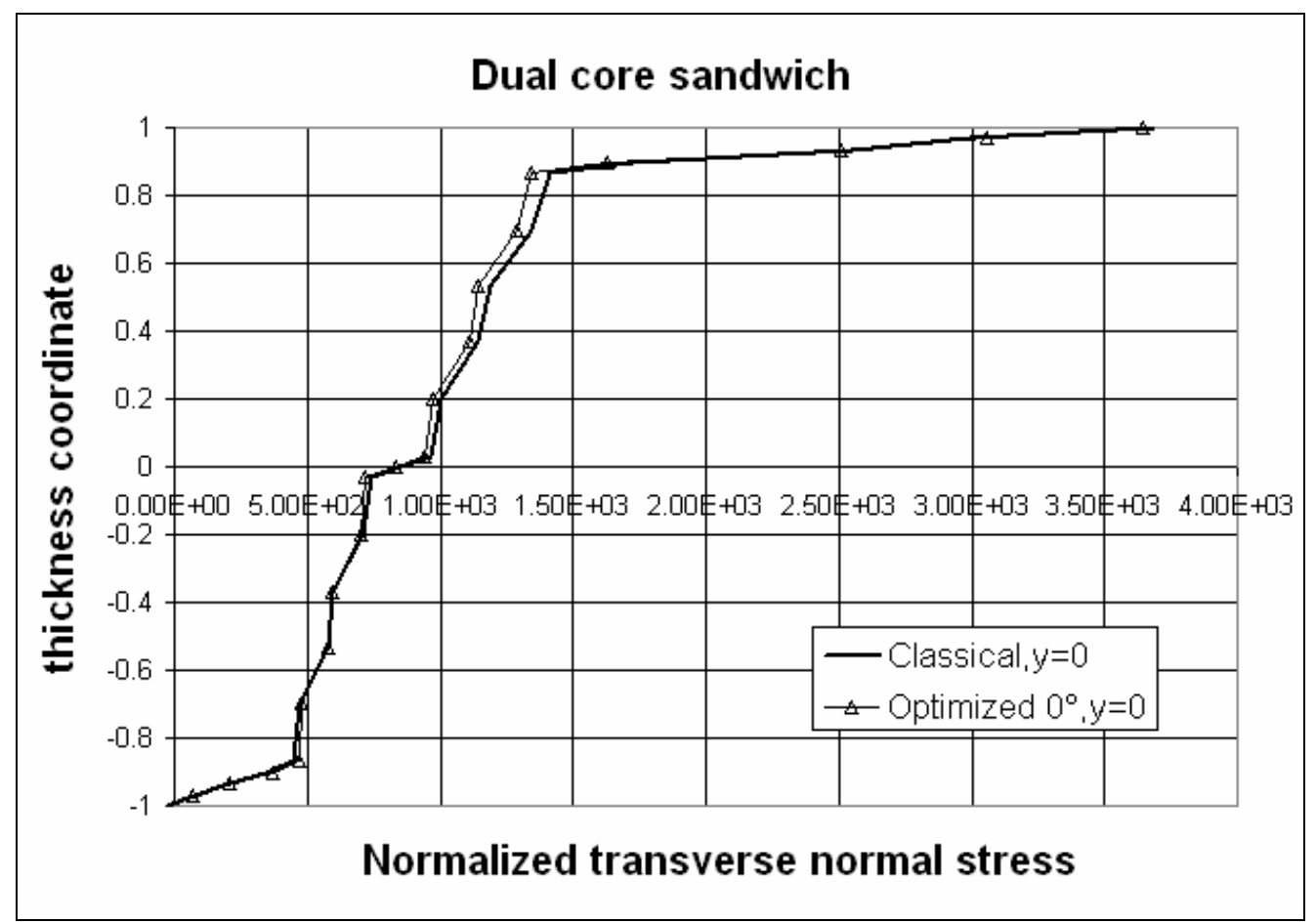

Fig.7. Through-the-thickness normalized transverse normal stress, at the impact point. Comparison between constant stiffness plies (Classical) and variable one (Optimized).

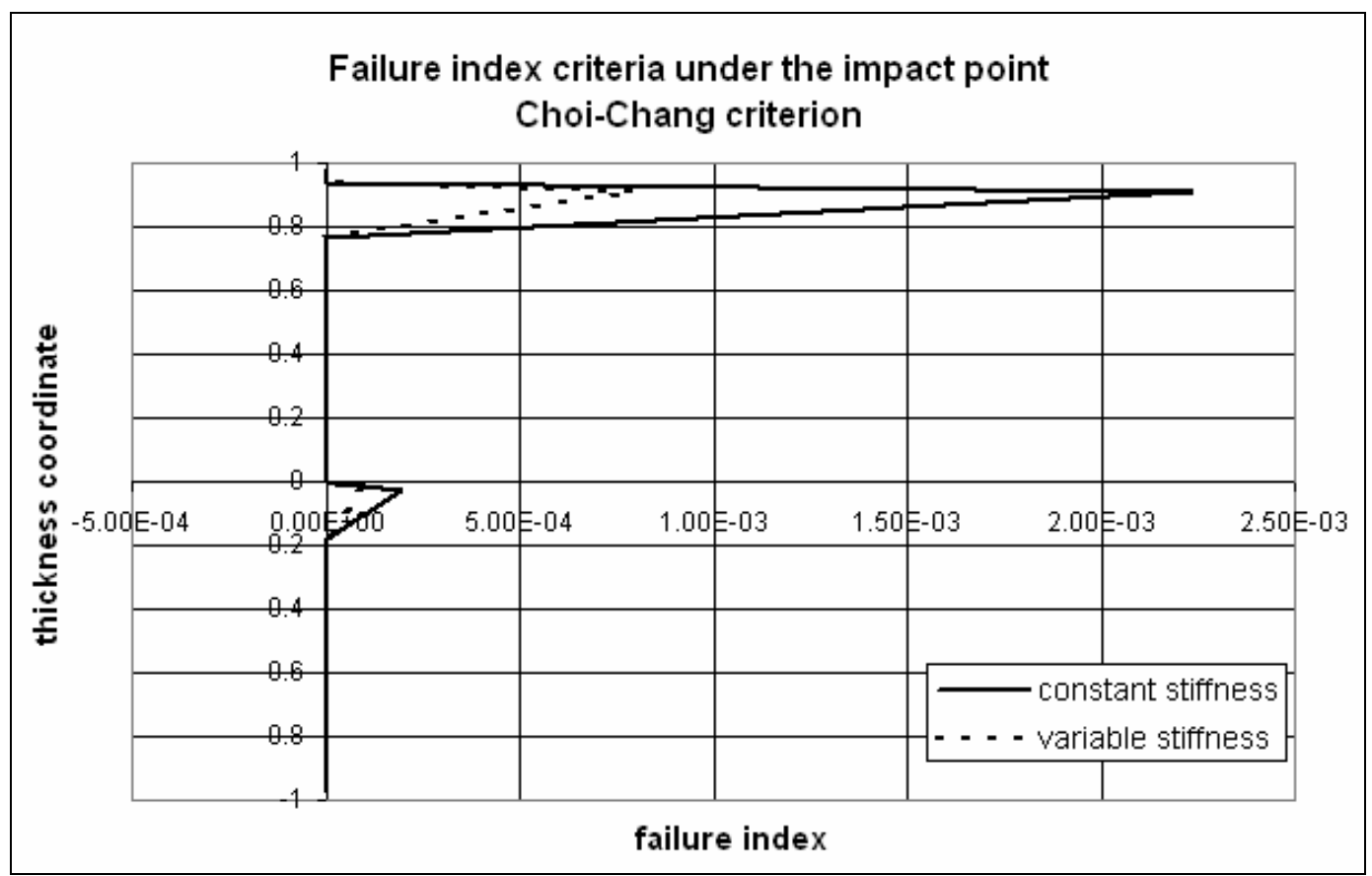

Fig.8. Failure index according to the Choi-Chang's criterion at the impact point. Comparison between constant and variable stiffness beams. 


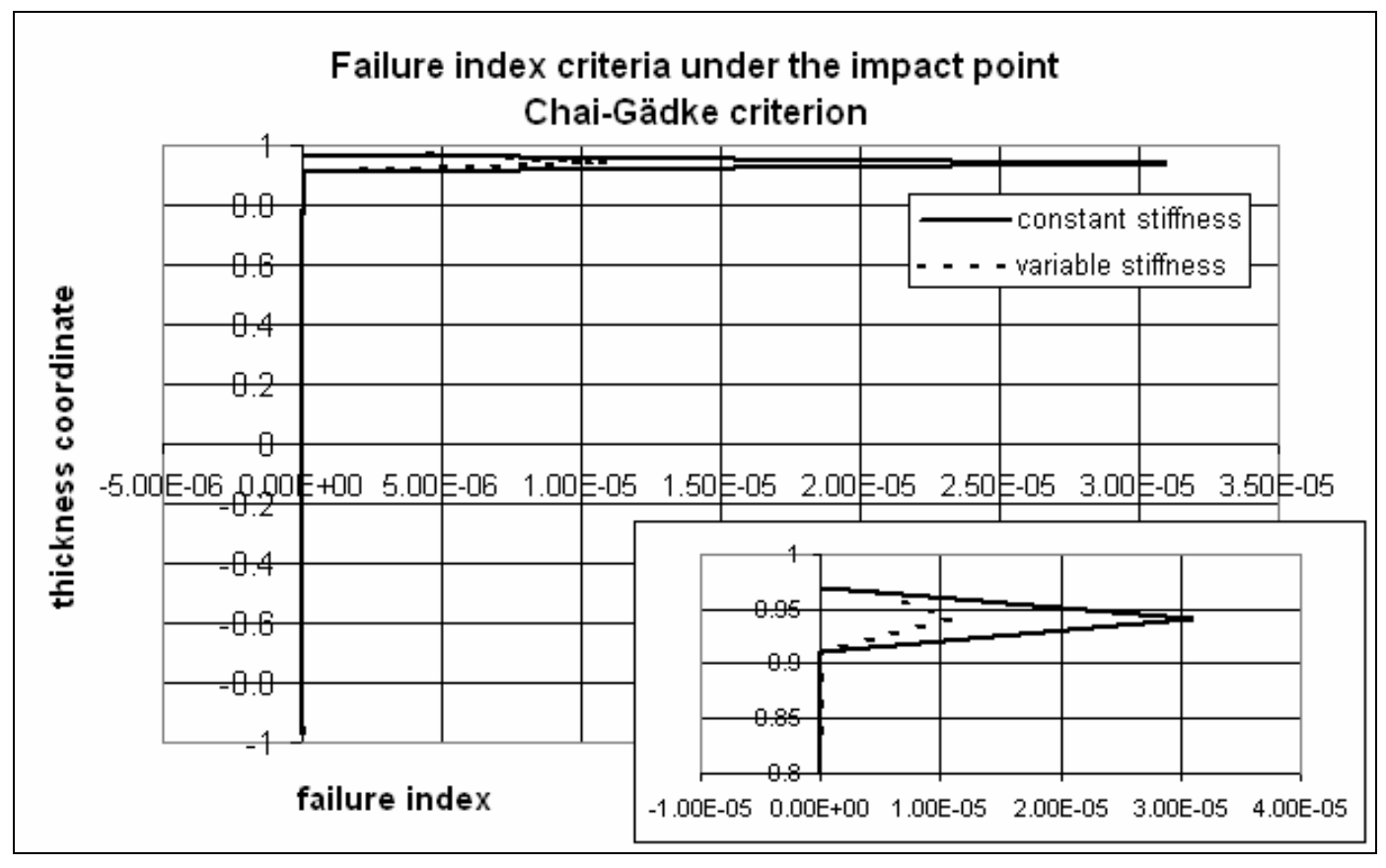

Fig.9. Failure index across according to the Chai-Gädke's criterion at the impact point. Comparison between constant and variable stiffness beams.

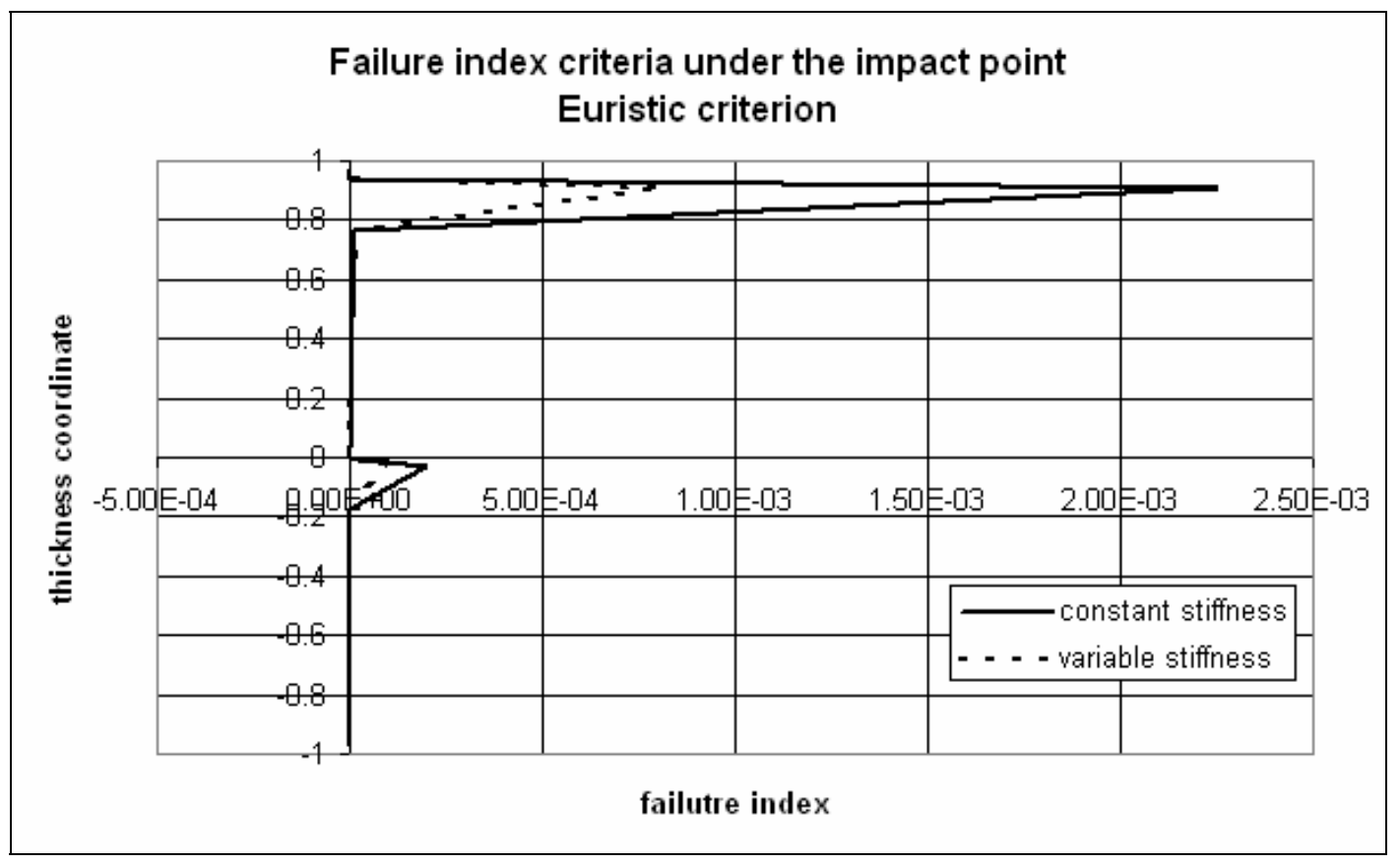

Fig.10. Failure index according to the euristic criterion described by Icardi [17] at the impact point. Comparison between constant and variable stiffness beam. 